\title{
Prevalencia de embarazo en adolescentes y factores asociados en el Hospital Luis F. Martínez. Cañar 2017
}

Prevalence of teenage pregnancy and associated factors at the Luis F. Martínez Hospital. Cañar 2017

口

Vázquez Bustos Wilson Patricio', Sempértegui Cárdenas

Pablo Xavier², Guamán Vásquez Ana Paulina ${ }^{3}$

\section{RESUMEN}

FECHA DE RECEPCIÓN: 27/08/2019 FECHA APROBACIÓN: 17/02/2021

Objetivo: determinar la prevalencia de embarazo en adolescentes y factores asociados en el Hospital Luis F. Martínez, de la provincia del Cañar, año 2017.

1. Hospital Homero Castanier Crespo. Medicina Familiar y Comunitaria. Consulta Externa. Cuenca - Ecuador

2. Hospital Homero Castanier Crespo. Pediatría. Hospitalización. Cuenca - Ecuador

3. Hospital de Especialidades José Carrasco Arteaga. Enfermería. Emergencia.

Cuenca - Ecuador

\section{Artículo Original Original Article}

DOI: https://doi.org/10.18537/RFCM.38.03.01

Correspondencia:

widovaz@hotmail.es

Dirección:

Avenida del Chofer 1-84

Código Postal:

010107

Teléfono:

0958612310

Cuenca - Ecuador

Metodología: estudio transversal realizado en 200 adolescentes mujeres. Se utilizó el test del APGAR Familiar y el cuestionario del Instituto Nacional de Estadísticas y Censos (INEC). Los datos se recolectaron en una encuesta y se tabularon en Epi Info 7. Se obtuvieron frecuencias, porcentajes, razón de prevalencia con su intervalo de confianza al 95\% así como Chi cuadrado con su valor $p$.

Resultados: el promedio de edad fue de 16.9 años; 52.5\% fueron mestizas, 41\% tuvieron instrucción secundaria incompleta, 51.5\% se encontraron en unión libre y eran amas de casa. La prevalencia de embarazo en adolescentes fue del 18\%. Los factores asociados a embarazo en la adolescencia fueron: vivienda distante al centro de salud (RP: 1.25, IC95\% 1.06-1.14, p=0.004), consumo de sustancias psicoactivas (RP 1.24, IC95\% 1.06-1.46, p=0.005), migración de uno de los padres (RP 1.28, IC95\% 1.10-1.49, p=0.002), deserción escolar (RP 1.65, IC95\% 1.34-2.03, p=0.000) y disfunción familiar (RP 1.66, IC95\% 1.5-2.21, $p=0.000)$.

Conclusiones: la prevalencia de embarazo en adolescentes en esta localidad es alta y está asociado con edad >15 años, vivienda distante al centro de salud, consumo de sustancias psicoactivas, migración de uno de los padres, deserción escolar y disfunción familiar.

Palabras clave: embarazo en adolescencia, factores de riesgo, factores culturales, factores socioeconómicos 


\section{ABSTRACT}

Objective: to determine the prevalence of pregnancy in adolescents and associated factors at the Luis F Martinez Hospital in Cañar province, in 2017.

Methodology: it is a cross-sectional study carried out in 200 female adolescents. The Family APGAR test and the questionnaire from the National Institute of Statistics and Censuses were used. Data was collected in a survey and it was tabulated in Epilnfo7. The frequencies, percentages, prevalence ratio with its 95\% confidence interval as well as Chi square with its $p$ value were obtained.

Results: the average age was 16.9 years; $52.5 \%$ were mestizo, $41 \%$ had incomplete secondary education, $51.5 \%$ were in free union and were housewives. The prevalence of teenage pregnancy was $18 \%$. The factors associated with adolescent pregnancy were: home distant from the health center (PR: 1.25, 95\% Cl 1.06-1.14, p: 0.004), consumption of psychoactive substances (PR 1.24, 95\% Cl 1.06-1.46, p: 0.005$)$, migration of one of the parents (PR 1.28, 95\% Cl 1.10-1.49, p: 0.002), school dropout (PR 1.65, 95\% Cl 1.34-2.03, p: 0.000 ) and family dysfunction (PR 1.66, 95\% Cl $1.5-2.21$, p: 0.000).

Conclusions: the prevalence of adolescent pregnancy in this locality is high and is associated with age >15 years, distant housing from the health center, consumption of psychoactive substances, migration of one of the parents, school dropout, and family dysfunction.

Key words: pregnancy in adolescence, risk factors, cultural factors, socioeconomic factors.

\section{INTRODUCCIÓN}

La Organización Mundial de la Salud (OMS) define la adolescencia como "el periodo de crecimientoy desarrollohumano queseproduce después de la niñez y antes de la edad adulta, entre los 10 y los 19 años" ${ }^{\prime 1}$. Se estima que 16 millones de adolescentes entre 15 a 19 años y aproximadamente 1 millón de niñas menores de 15 años dan a luz cada año, la mayoría en países de ingresos bajos y medianos ${ }^{2}$. Las complicaciones durante el embarazo y el parto son la segunda causa de muerte entre las jóvenes de 15 a 19 años en todo el mundo², y el embarazo en la adolescencia se ha relacionado con una importante carga de morbilidad ${ }^{3}$.

La familia desempeña un papel fundamental en la sociedad ${ }^{4}$ y la funcionalidad familiar es un determinante muy importante en la consolidación del comportamiento del adolescente ${ }^{5,6}$. El embarazo en la adolescente desarrolla variaciones radicales y nuevas adaptaciones como la maternidad temprana, adaptación psicosocial laboral y readaptación intrafamiliar ${ }^{4}$. La funcionalidad familiar es la capacidad de adaptación de sistema familiar para afrontar y superar las etapas del ciclo vital y las crisis que presenta ${ }^{5}$. Entre los factores estudiados como causa de embarazo en adolescentes destacan: edad, falta de acceso a educación y servicios de salud reproductiva, pobreza, violencia y coacción sexual, una educación sexual inadecuada, migración, distancia de vivienda al centro de salud7,8-15.

En Estados Unidos la prevalencia de embarazo en adolescente es de 12.8\%, en El Salvador 25\% y países africanos cerca del $45 \%{ }^{16}$, mientras que en Cuenca-Ecuador se ha estimado en el $11.6 \%{ }^{17}$, dando cuenta de la gran variabilidad existente entre regiones; sin embargo, no se han realizado estudios de prevalencia de embarazo en adolescentes en la ciudad de Cañar, así como tampoco de los factores asociados al mismo, resultando importante determinar 
la influencia de factores tales como: edad, condición socio-económica, etnia, distancia de la vivienda al centro de salud, conocimiento en salud sexual y reproductiva, funcionalidad familiar, consumo de sustancias psicoactivas, estabilidad y migración parental, antecedente de embarazos previos y deserción escolar en el embarazo de la adolescente.

\section{METODOLOGÍA}

Se realizó un estudio descriptivo, transversal. El universo fueron todas las mujeres hospitalizadas (por cualquier causa) en el hospital Luis F. Martínez dela provincia del Cañar en el año 2017. La muestra fue probabilística y se calculó en el programa Epi Info versión 7, con la opción de cálculo para un estudio descriptivo (population survey) utilizando las siguientes restricciones muestrales: tamaño de la población: 413, frecuencia esperada: 73.5\% (basado en frecuencia de adolescentes embarazadas de acuerdo a datos de estadística de egresos del Hospital Luis F. Martínez del 2016), límites de confianza 5\%, intervalo de confianza de 95\%, obteniéndose 174 casos a los cuales se añadió un 15\% por posibles pérdidas dando un total de 200 adolescentes.

Se incluyeron únicamente mujeres entre 10 y 19 años ingresadas en el hospital que aceptaron su participación mediante asentimiento informado y que contaban con la presencia de un acompañante legal para firmar el consentimiento informado; fueron excluidos los casos con problemas mentales que dificultaban la comunicación.

La información se recolectó mediante una encuesta estructurada y la observación simple de los datos de las historias clínicas. Para determinar la funcionalidad familiar se aplicó el cuestionario de Apgar Familiar validado previamente por Smilkestein ${ }^{18}$, dicho instrumento consta de cinco preguntas que califican: apoyo, participación, crecimiento, afecto y recreación, para cada uno de los cuales se califica como casi siempre (2 puntos), algunas veces (1 punto) y casi nunca (0 puntos), considerándose familia funcional los que obtienen puntajes de 7 a 10, disfuncionalidad moderada de 4 a 6 y disfuncionalidad severa de 0 a 3 puntos. La condición socio-económica se evaluó mediante la encuesta de Estratificación del Nivel Socio-económico del INEC ${ }^{19}$. Para la variable conocimiento en salud sexual y reproductiva se usó el formulario de Verleye: "Durex Network and Trendwolves" previamente validado en su versión al español20.

Los datos fueron tabulados en el programa Epi Info versión 7, de las variables cuantitativas tales como: edad y distancia al centro de salud (en minutos), se obtuvieron: medias, desviación, valores máximos y mínimos. De las variables cualitativas tales como: autoidentificación étnica, parroquia, ocupación, nivel de instrucción, estado civil, embarazo previo, deserción escolar, pérdida reciente de personas significativas, migración del padre o madre, vivienda distante al centro de salud, conocimiento en salud sexual y reproductiva, consumo de sustancias psicoactivas y estado civil de los padres; se obtuvieron frecuencias y porcentajes. Para el cálculo de la prevalencia de embarazo en adolescentes se consideró el número de adolescentes embarazadas respecto al total de embarazos documentados en el mismo período de tiempo. Para determinar la asociación entre embarazo y las variables mencionadas en el grupo de adolescentes, los datos se dicotomizaron en tablas tetracóricas. Se calcularon: razón de prevalencia (RP) con su intervalo de confianza al 95\% y los valores $p$ mediante prueba de Chi-cuadrado con test de Fisher.

\section{RESULTADOS}

Se incluyeron 200 participantes con una edad promedio 16.9 años (DS 1.8). El 52\% se autoidentificó como mestiza, 46.5\% como indígena 
y $1 \%$ como blanca. El $41 \%$ tuvo una instrucción secundaria incompleta, $31.5 \%$ primaria incompleta, $11 \%$ primaria completa, $8.5 \%$ secundaria completa, $6.5 \%$ fueron analfabetas y $1.5 \%$ tuvieron instrucción técnica, el resto de variables sociodemográficas se detallan en la Tabla $N^{\circ} 1$.

\section{Tabla $\mathrm{N}^{\circ} 1$}

Distribución de adolescentes ingresadas en el Hospital Luis F. Martínez de acuerdo a variables socio-demográficas. Cañar 2017

\begin{tabular}{lrr}
\hline \multicolumn{1}{c}{ Variable } & $\mathbf{n}$ & $\%$ \\
\hline Edad & 16 & 8.0 \\
$\quad$ Adolescente temprano $(<15)$ & 184 & 92.0 \\
$\quad$ Adolescente tardío $(\geq 15)$ & & \\
Estado civil & 21 & 10.5 \\
$\quad$ Casada & 71 & 35.5 \\
Soltera & 103 & 51.5 \\
Unión libre & 1 & 0.5 \\
Viuda & 4 & 2.0 \\
Divorciada & & \\
Ocupación & 103 & 51.5 \\
Quehaceres domésticos & 7 & 3.5 \\
Trabajadora pública & 6 & 3.0 \\
Trabajadora privada & 14 & 7.0 \\
Negocio propio & 40 & 20.0 \\
Estudiante & 30 & 15.0 \\
Ninguna & & \\
Residencia & 81 & 40.5 \\
Cañar & 9 & 4.5 \\
Chontamarca & 4 & 2.0 \\
Gualleturo & 6 & 3.0 \\
Juncal & 3 & 1.5 \\
Zhud & 6 & 3.0 \\
General Morales & 5 & 2.5 \\
Ducur & 2 & 1.0 \\
Chorocopte & 6 & 10.0 \\
Ingapirca & 6 & 3.0 \\
Honorato Vázquez & 58 & 29.0 \\
Otra & & \\
\hline
\end{tabular}

El $76.7 \%$ de adolescentes cursaban su primer embarazo, el $20.7 \%$ segundo y $2.7 \%$ tercero. La prevalencia de embarazo en adolescentes fue de $18 \%$.

La tasa de embarazo en menores de 15 años fue de $25 \%$ vs $79 \%$ en $\geq 15$ ( $p=0.000)$; de igual manera fue mayor en adolescentes de condición socio-económica baja respecto a otras condiciones ( $78 \%$ vs $50 \%$ ) ( $p=0.006)$; las pacientes con una vivienda distante al centro de salud tuvieron una frecuencia mayor de embarazo comparadas con aquellas con viviendas cercanas ( $84 \%$ vs $67 \%)(p=0.004)$; el consumo de sustancias psicoactivas mostró también un mayor porcentaje de embarazos con respecto a aquellas sin consumo (84\% vs $67 \%)(p=0.005)$; como también ocurrió en adolescentes con migración de alguno de los padres ( $86 \%$ vs $67 \%$ ) ( $p=0.002)$; las pacientes con deserción escolar tuvieron embarazos en el $89 \%$ vs el $54 \%$ en aquellas sin deserción 
$(\mathrm{p}=0.000)$; el antecedente de pérdida de alguna persona significativa mostró también una mayor frecuencia de embarazos (84\% vs $67 \%)(p=0.003)$; finalmente el antecedente de embarazo previo tuvo una frecuencia de embarazos de $98 \%$ vs $67 \%$ en el grupo sin este antecedente $(p=0.000)$. El resto de variables estudiadas no mostraron asociación significativa (Tabla $N^{\circ} 2$ ).

\section{Tabla $N^{\circ} 2$}

Distribución de adolescentes ingresadas en el Hospital Luis F. Martínez de acuerdo a embarazo y factores asociados. Cañar 2017

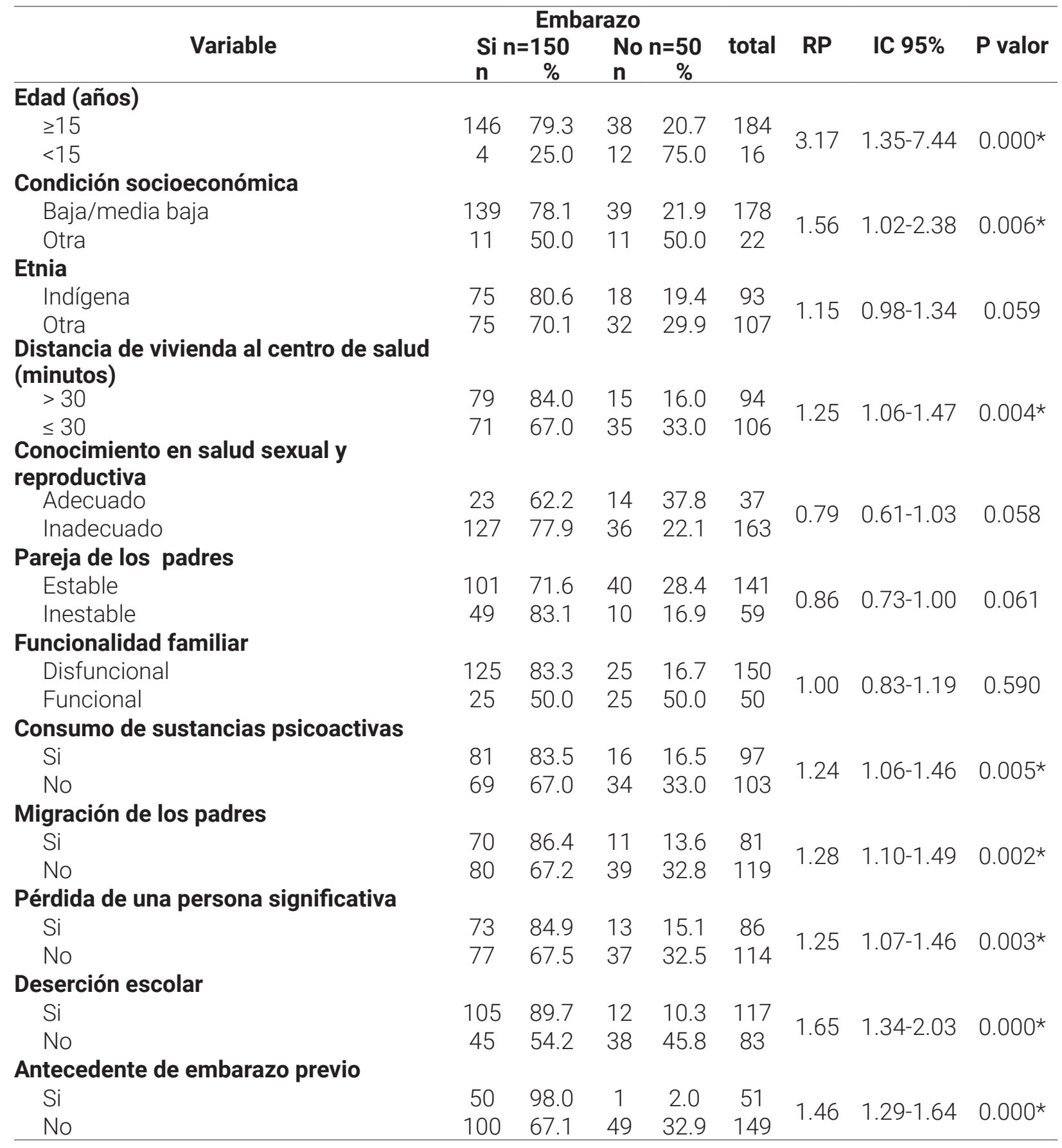

*existe diferencia estadísticamente significativa 


\section{DISCUSIÓN}

En el presente estudio la prevalencia de embarazo en adolescentes fue de $18 \%$, cifra similar al reporte oficial del INEC con un $17 \%$ de embarazo entre los 15 y 19 años ${ }^{21}$, y al reportado en África con un $18.8 \%{ }^{22}$; sin embargo, al considerar únicamente a las adolescentes hospitalizadas observamos una tasa de embarazo del $25 \%$ en menores de 15 años y 79\% en mayores de esta edad, prevalencia muy superior a la publicada, lo que probablemente obedece a que la mayor parte de adolescentes que acuden al Hospital Luis F. Martínez lo hacen para atención de parto o sus complicaciones y debido también a que no se incluyeron en este estudio pacientes sanas, por lo cual no permite el cálculo de la prevalencia real a nivel comunitario; por otro lado, ya se ha publicado previamente el riesgo de embarazo adolescente asociado a una mayor edad como lo demuestra el estudio de Pinzón et al. ${ }^{9}$, donde a mayor edad existió mayor frecuencia de embarazos (OR: 0.60) lo que también fue demostrado en el presente estudio con una frecuencia mayor de embarazo en > de 15 años $(p=0.000)$.

La condición socioeconómica ha sido ampliamente estudiada, Chung et al. ${ }^{23}$ asevera que las adolescentes con un nivel económico bajo, pobreza y menores ingresos tienen más probabilidades de quedar embarazadas que sus contrapartes. En otro estudio en Malasia, Omar et al. ${ }^{13}$, demostró que la tasa de embarazo en adolescentes en el grupo de ingresos menores a \$153 alcanzó un 79\%, similar al 78\% encontrado en el presente estudio en el grupo de condición socioeconómica baja o mediobaja, esta prevalencia alta probablemente sea un reflejo de la disparidad existente en el acceso a servicios de salud y educación, así como la influencia de variables culturales cuyo análisis debería reforzarse en estudios posteriores.
Respecto a la etnia, no se pudo demostrar asociación estadística con embarazo adolescente, esto concuerda con el estudio de Pinzón et al. ${ }^{9}$, donde la etnia indígena no mostró asociación significativa $(p=0.349)$; sin embargo, este hallazgo debería analizarse con cautela dado que es lógico suponer que en ciertas minorías étnicas el inicio de vida sexual así como el matrimonio, es una práctica cultural aceptada a edades tempranas, en el presente estudio probablemente no pudo ser visibilizado, dada la influencia cultural del mestizaje en las poblaciones estudiadas. Sería recomendable investigar las desigualdades étnicas en la atención de la salud ${ }^{24}$

Es lógico suponer que vivir cerca de un establecimiento de salud permite un mayor acceso al servicio, lo cual resulta trascendental en materia de salud sexual y reproductiva, este hecho fue estudiado por Olsen et al. ${ }^{12}$ en Reino Unido mediante un sistema de georeferenciación en el cual se determinó que los jóvenes tienen más probabilidades de acudir a un centro médico para atención en salud sexual y reproductiva, si dicho centro se encontraba más cerca de su domicilio (OR: 2.1, IC95\%: 1.5-3.0); esto podría explicar porqué en el presente estudio la tasa de embarazo en adolescentes fue superior en aquellas que vivían más alejadas del centro de salud.

Aunque la tasa de embarazo en adolescentes fue mayor en el grupo con conocimiento inadecuado en salud sexual y reproductiva, la diferencia no fue significativa respecto a aquellas con conocimiento adecuado $(p=0.05)$; sin embargo, esta variable ha sido demostrada en estudios previos como un factor de riesgo importante, como lo publica Rodríguez et al ${ }^{14}$, demostrando un OR de 3.3 para embarazo adolescente en aquellas con falta de conocimiento respecto a complicaciones del embarazo. 
Omar et al. ${ }^{13}$, demostró que el consumo de sustancias representa un factor de riesgo para embarazo en adolescentes $(p=0.001)$, así como Chung et al. ${ }^{23}$ asevera que el consumo de sustancias aumenta la probabilidad de quedar embarazada, esto concuerda con los hallazgos encontrados en el presente estudio; queda por determinarse sin embargo, si el consumo propiamente es el que motiva las prácticas sexuales inseguras o coinciden en el contexto de otras variables no estudiadas en función del bienestar psicológico del adolescente, lo cual podría reflejarse en otros hallazgos de la presente investigación tales como una mayor tasa de embarazos en el grupo con pérdidas de una persona significativa, migración de alguno de los padres o deserción escolar.

Una revisión sistemática reciente realizada por Fellmeth et al. ${ }^{10}$, puntualiza sobre la falta de estudios existentes respecto al riesgo de embarazo adolescente en función de la migración parental, por lo cual se considera que la asociación aquí reportada representa una contribución importante al estado del arte.

Finalmente, dado que los estudios transversales constituyen el nivel más débil de evidencia para demostrar causalidad, se considera necesaria la realización de futuras investigaciones con diseños analíticos y muestras más grandes que permitan confirmar las asociaciones aquí encontradas; así como también, el estudio de otras variables que ayuden al entendimiento del embarazo en adolescentes para el diseño de políticas públicas en materia de prevención.

\section{CONCLUSIONES}

La prevalencia de embarazo en adolescentes en esta localidad es alta y está asociado con edad >15 años, condición socioeconómica baja, vivienda distante al centro de salud, consumo de sustancias psicoactivas, migración parental, pérdida de una persona significativa, deserción escolar y antecedente de embarazo previo.

\section{ASPECTOS BIOÉTICOS}

El estudio se realizó de acuerdo a lo señalado en la declaración de Helsinki y fue aprobado previamente por el Comité de Bioética de la Facultad de Ciencias Médicas de la Universidad de Cuenca-Ecuador. A pesar de no ser un estudio de intervención, dado que se trabajó con encuestas personales, se obtuvo en todos los casos el asentimiento informado de las adolescentes, así como el consentimiento informado de su representante legal para su participación, no se incluyeron datos de identificación y se mantuvo en todo momento la confidencialidad de la información proporcionada.

\section{INFORMACIÓN DE LOS AUTORES}

Vázquez Bustos Wilson Patricio. Médico. Especialista en Medicina Familiary Comunitaria. Hospital Homero Castanier Crespo. Medicina Familiar y Comunitaria. Consulta Externa. Cuenca-Azuay-Ecuador.

e-mail:widovaz@hotmail.es

ORCID:https://orcid.org/0000-0003-3091-4906

Sempértegui Cárdenas Pablo Xavier. Médico. Magíster en Investigación de la Salud. Hospital Homero Castanier Crespo. Pediatría. Hospitalización. Cuenca-Azuay-Ecuador.

e-mail: troverospa@gmail.com

ORCID:https://orcid.org/0000-0001-7325-3082

Guamán Vásquez Ana Paulina. Licenciada en Enfermería. Hospital de Especialidades José Carrasco Arteaga. Enfermería. Emergencia. Cuenca-Azuay-Ecuador.

e-mail: Icda.paulinaguamanvasquez@gmail.com

ORCID:https://orcid.org/0000-0003-2661-9066

\section{CONTRIBUCIÓN DE LOS AUTORES}

VBWP: Concepción y diseño del estudio. Tabulación, análisis e interpretación de los datos. Redacción y revisión crítica del manuscrito. Aprobación del informe final. 
SCPX: Diseño del estudio, revisión bibliográfica, revisión crítica del manuscrito. Elaboración y aprobación del documento final.

GVAP: Concepción y diseño del estudio. Tabulación, análisis e interpretación de los datos. Redacción y revisión crítica del manuscrito. Aprobación del informe final.

\section{CONFLICTO DE INTERESES}

Los autores declaran la no existencia de conflicto de intereses

\section{FUENTES DE FINANCIAMIENTO}

Autofinanciado.

\section{REFERENCIAS BIBLIOGRÁFICAS}

1. Organización Mundial de la Salud. Salud de la madre, el recién nacido, del niño y del adolescente. Desarrollo en la adolescencia [Internet]. 2019 [citado 21 de agosto de 2019]. Disponible en: https://www.who. int/maternal_child_adolescent/topics/ adolescence/dev/es/

2. Organización Mundial de la Salud. El embarazo en la adolescencia [Internet]. [actualizado 31 Ene 2020; citado 13 Ago 2020]. Disponible en: https://www.who. int/es/news-room/fact-sheets/detail/ adolescent-pregnancy

3. Fernandes de Azevedo W, Baffi Diniz M, Borges Da Fonseca E, Ricarte de Azevedo L, Braz Evangelista C. Complications in adolescent pregnancy: systematic review of the literatura. Einstein. 2015; 13 (4): 61826. doi: 10.1590/S1679-45082015RW3127

4. Valladarez-Loján JV. Factores de riesgo familiar que predisponen al embarazo en las adolescentes del Hospital Isidro Ayora periodo abril-septiembre 2015. [B.S. thesis].
2016. Disponibleen:https://dspace.unl.edu. ec/jspui/bitstream/123456789/17154/1/ Jessica\%20\%20Ver\%c3\%b3nica\%20 Valladarez\%20Loj\%c3\%a1n.pdf

5. Adebayo AM, Ajuonu EJ, Betiku BO. Family functionality and parental characteristics as determinants of sexual decision-making of in-school youths in a semi-urban area of Southwest Nigeria. (2016). International Journal of Adolescent Medicine and Health, 28 (4): 413-18. doi: https://doi. org/10.1515/ijamh-2015-0029

6. Higuita-Gutiérrez LF, Cardona-Arias JA. Percepción de funcionalidad familiar en adolescentes escolarizados en instituciones educativas públicas de Medellín (Colombia), 2014. 2016. Rev. CES Psicol., 9 (2): 167-178. doi: http://dx.doi. org/10.21615/cesp.9.2.11

7. Jiménes $M$, Romero $E$, García $M$, Bravo C. Resultados obstétricos y perinatales de las gestantes adolescentes atendidas en el Hospital Central de la Defensa Gómez Ulla [Internet]. 2017 [citado 21 de agosto de 2019]. doi: 10.4321/s188785712017000300004.Disponible en: http:// scielo.isciii.es/pdf/sm/v73n3/1887-8571sm-73-03-00158.pdf

8. Salinas-Mulder S, Castro-Mantilla MD, Fernández-Ovando C. Vivencias y relatos sobre embarazo en adolescentes. Una aproximación a los factores culturales, sociales y emocionales a partir de un estudio en seis países de la región. Informe final. UNICEF. Agosto 2014. Disponible en: https://www.unicef. org/republicadominicana/embarazo_ adolescente_5_0_(2).pdf 
9. Pinzón-Rondón AM, Ruiz-Sternberg AM, Aguilera-Otalvaro PA, Abril-Basto PA. Factores asociados al inicio de vida sexual y al embarazo adolescente en Colombia. Estudio de corte transversal. Rev. chil. obstet. ginecol. 2018; 83(5): 487-99. doi: $10.4067 / s 0717-75262018000500487$

10. Fellmeth G, Rose-Clarke K, Zhao Ch, Busert L, Zheng $Y$, Massazza A, et al. Health impacts of parental migration on left-behind children and adolescents: a systematic review and meta-analysis. The Lancet 2018, 392 (10164): 2567-82. doi: 10.1016/ S0140-6736(18)32558-3

11. Miller B, Benson B. Galbraith K. Family Relationships and Adolescent Pregnancy Risk: A Research Synthesis. Developmental review, 2001, 21 (1): 1-38. doi: 10.1006/ drev.2000.0513 Disponible en: https:// www.sciencedirect.com/science/article/ abs/pii/S0273229700905136

12. Olsen J, Cook P, Forster S, Phillips-Howard $P$. Accessibility of sexual health services in teenage sexual health service users: local area geospatial analysis. Journal of Public Health 2012. 34 (3): 438-46. doi:10.1093/ pubmed/fdr120

13. Omar K, Hasim S, Muhammad A, Jaffar A. Adolescent pregnancy outcomes and risk factors in Malaysia. OMAR, Khairani, et al. Adolescent pregnancy outcomes and risk factors in Malaysia. International Journal of Gynecology \& Obstetrics. 2010. 111 (3): 220-3. doi: 10.1016/j.ijgo.2010.06.023. Disponible en: https://obgyn.onlinelibrary. wiley.com/doi/abs/10.1016/j. ijgo.2010.06.023
14. Rodríguez-Rodríguez N, Cala-Bayeux A, Nápoles-Pérez JL, Milán-Arenado Y, AguilarTito M. Factores de riesgo asociados al embarazo en adolescentes. Revista Información Científica. 2017. 96 (1): 29-37. Disponible en: http://www.revinfcientifica. sld.cu/index.php/ric/article/view/27/780

15. Yakubu I, Salisu W. Determinants of adolescent pregnancy in sub-Saharan Africa: a systematic review. Reproductive Health. 2018. 15 (1): 15. doi: 10.1186/ s12978-018-0460-4

16. León $P$, Minassian $M$, Borgoño $R$, Bustamante F. Embarazo adolescente. Rev Ped Elec. 2008. 5(1): 42-51.

17. Guevara-Pérez WO. Prevalencia de embarazo en adolescentes y factores psicosociales asociados en colegios de Cuenca Ecuador 2012 [Internet]. 2013. Disponible en: http://dspace.ucuenca. edu.ec/bitstream/123456789/4006/1/ MEDG041.pdf

18. Suárez MA, Alcalá-Espinoza M. Apgar Familiar: una herramienta para detectar disfunción familiar. Rev Med La Paz. 2014. 20(1): 53-7. Disponible en: http://www. scielo.org.bo/pdf/rmcmlp/v20n1/v20n1_ a10.pdf

19. INEC. Encuesta de Estratificación del nivel Socioeconómico [Internet]. 2010 [citado 21 de agosto de 2019]. Disponible en: https://www.ecuadorencifras.gob. ec//documentos/web-inec/Estadisticas_ Sociales/Encuesta_Estratificacion_ Nivel_Socioeconomico/Cuestionario_ Estratificacion.pdf 
20. Verleye, G. Reproductive Healths Knowledge, attitudes, and practices a european youth study protocol. 2009. Disponible en: http://www.durexnetwork. org/SitecollectionDocuments / Research\%20Protocol1.pdf

21. Villacis B, Carrillo D. País atrevido: la nueva cara sociodemográfica del Ecuador. Edición especial revista Analitika. Instituto Nacional de Estadística y Censos (INEC). Quito - Ecuador. 2012. Disponible en: https://www.ecuadorencifras.gob.ec/wpcontent/descargas/Libros/Economia/ Nuevacarademograficadeecuador.pdf

22. Kassa GM, Arowojolu AO, Odukogbe AA, Yalew AW. Prevalence and determinants of adolescent pregnancy in Africa: a systematic review and Meta-analysis. Reprod Health. 2018; 15 (1): 195. Publicado el 29 de noviembre de 2018. doi: 10.1186 / s12978-018-0640-2

23. Chung HW, Kim EM, Lee JE. Comprehensive understanding of risk and protective factors related to adolescent pregnancy in low- and middle-income countries: A systematic review. J Adolesc. 2018; 69: 180-188. doi: 10.1016/j.adolescence.2018.10.007

24. Paulino NA, Vázquez MS, Bolúmar $F$. Indigenous language and inequitable maternal health care, Guatemala, Mexico, Peru and the Plurinational State of Bolivia. Bull World Health Organ. 2019; 97(1): 5967. doi: 10.2471/BLT.18.216184 